\title{
P 272 IDENTIFICATION OF THE MOST COMMONLY PRESCRIBED OPIOID FOR ADMINISTRATION BY CONTINUOUS SUBCUTANEOUS INFUSION
}

Andrew Dickman. Marie Curie Palliative Care Institute, Liverpool, Liverpool, UK

10.1136/bmjspcare-2014-000654.313

Background Continuous subcutaneous infusions (CSCI) are commonly used to maintain symptom control once the oral route is no longer available. In 2004, there was a worldwide shortage of diamorphine and most centres adopted morphine as the opioid of choice. Diamorphine has since become available again, but it is more expensive than morphine. A national survey of UK practice performed in summer 2004 identified diamorphine as the most commonly prescribed opioid for administration by CSCI. No survey of national practice has been performed since.

Aims To identify the most commonly prescribed opioid for administration by CSCI in the UK and to compare with local practice.

Method UK pharmacists and pharmacy technicians were invited to complete an internet-based national survey of current practice. For each CSCI, participants recorded information about constituent drugs, doses, diluent, total volume, duration of administration and visual appearance. A sub-analysis of the data was performed in order to identify the frequency of opioid prescribing. The results were compared to those obtained from local practice.

Results A total of 2000 CSCIs were recorded in the national survey by 35 centres distributed across the UK. Morphine was the most commonly prescribed opioid, occurring in $28 \%$ of CSCIs. Oxycodone was the second most frequently prescribed opioid, followed by alfentanil and diamorphine. Analysis of local practice identified morphine as the most commonly prescribed opioid, with alfentanil being the second, followed by oxycodone and diamorphine.

Conclusion Morphine has been shown to be the most commonly prescribed opioid for administration by CSCI. 